\title{
Lyoluminescence: A theoretical approach
}

\author{
Amit Kr. Chattopadhyay \\ Department of Theoretical Physics, Indian Association for the Cultivation of Science, Jadavpur, 700032 Calcutta, India \\ G. S. Mahapatra \\ Department of Spectroscopy, Indian Association for the Cultivation of Science, Jadavpur, 700032 Calcutta, India \\ Pinaki Chaudhury \\ Department of Physical Chemistry, Indian Association for the Cultivation of Science, Jadavpur, 700032 Calcutta, India
}

(Received 30 March 1999; revised manuscript received 8 June 1999)

\begin{abstract}
When strongly energized halide or organic crystals are dissolved in a liquid solvent (like water), light is emitted as a result of a recombination process. This phenomenon is called lyoluminescence. The emitted light intensity, called the lyoluminescent intensity, depends on a class of factors like radiation dose, probability of radiative recombination, rate of dissolution in the solvent, etc. Combining some of these numerous effects we develop a nonlinear differential equation and analyze it by a dynamical system analysis as well as by exact numerical integration. The corresponding plot of the theoretical lyoluminescent intensity versus time graph, called the glow curve (Fig. (1)), matches very well with the shape of the experimental curve (Fig. (2)) for a vast range of characteristic values of the controlling parameters.
\end{abstract}

\section{INTRODUCTION}

Several organic substances and halide crystals produce a glow when lengthy exposure to high-energy radiation like $\mathrm{x}$ rays, $\gamma$ rays, etc. is followed by immersion in a liquid solvent. This glow is called the lyoluminescent (LL) glow. The first known instance of observation of lyoluminescence was due to Wiedemann and Schmidt, ${ }^{1}$ who observed lyoluminescence in glucose. After exposure to ionizing radiation and the decay of initial glow transients, dissolution of glucose in water at room temperature produced a glow which initially increased with time and then gave a steady illumination after about a minute. Later Ahnstrom and Ehrenstein ${ }^{2}$ observed that unirradiated glucose did not show any glow on dissolution. Extending their earlier work, Ahnstrom and Ehrenberg $^{3,4}$ subsequently used the glow to determine the density of $F$ centers in an inorganic sample as a function of the incident $\gamma$ dose. Lelievre and Adloff ${ }^{5}$ measured essentially the same quantity with halide crystals. In an attempt to increase this LL radiation, it was observed that LL of glucose was enhanced when the $p \mathrm{H}$ of the solution was increased ${ }^{6,7}$. The LL intensity was found to be proportional to the amount of solute dissolved and also proportional to the radiation dose $^{6}$ to which the sample was exposed. Although variation of these different parameters proved to have a non-negligible effect on LL, attempts towards determining the quantitative dependence of LL intensity on these parameters were mainly due to Burns and Williams ${ }^{7}$ and Mittal. ${ }^{8}$

Notwithstanding continuous experimental investigations in this front, a theoretical effort towards the quantitative estimation of LL from organic substances was due to Chatterjee, Sur, and Roy. ${ }^{9}$ Their approach centered around the developement of a rate equation from phenomenological considerations using mechanisms already suggested by Ettinger and Puite, ${ }^{10,11}$ Russel, and Vassil'ev. ${ }^{12,13}$

In the present paper we attempt to develop a theoretical framework for the variation of LL intensity of alkali halides with time, the basic mechanism being that due to Atari. ${ }^{14} \mathrm{We}$ consider only some of the major controlling parameters elaborated in Ref. 14 in the course of developement of our rate equation and compare our theoretical results with known experimental data.

\section{MECHANISM OF LYOLUMINESCENCE}

According to the mechanism of Atari, ${ }^{14}$ when an energized halide crystal is dissolved in solution, the entire process of LL takes place in two stages: one in the solid phase of the sample when it is irradiated with an $\mathrm{x}$ ray or a $\gamma$ ray and the other in the liquid phase when it undergoes dissolution. When an alkali halide crystal is dissolved in water, two effects occur simultaneously; an electron is released from an $F$ center and a hydrated electron $\left(e_{a q}^{-}\right)$is formed. The large quenching effect of the hydrated electron acceptors indicates that the released $F$ center undergoes hydration before its recombination with a $V_{2}$ center. $^{4,17}$ The rapid recombination of the hydrated electron with a $V_{2}$ center at the water-solid interface gives rise to the luminescence. Schematically the process reads as follows:

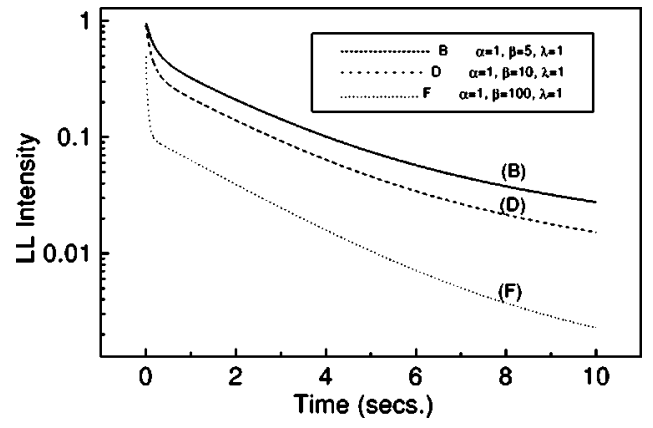

FIG. 1. Theoretical glow curves (semilog) for different $\alpha, \beta$, and $\lambda$. 


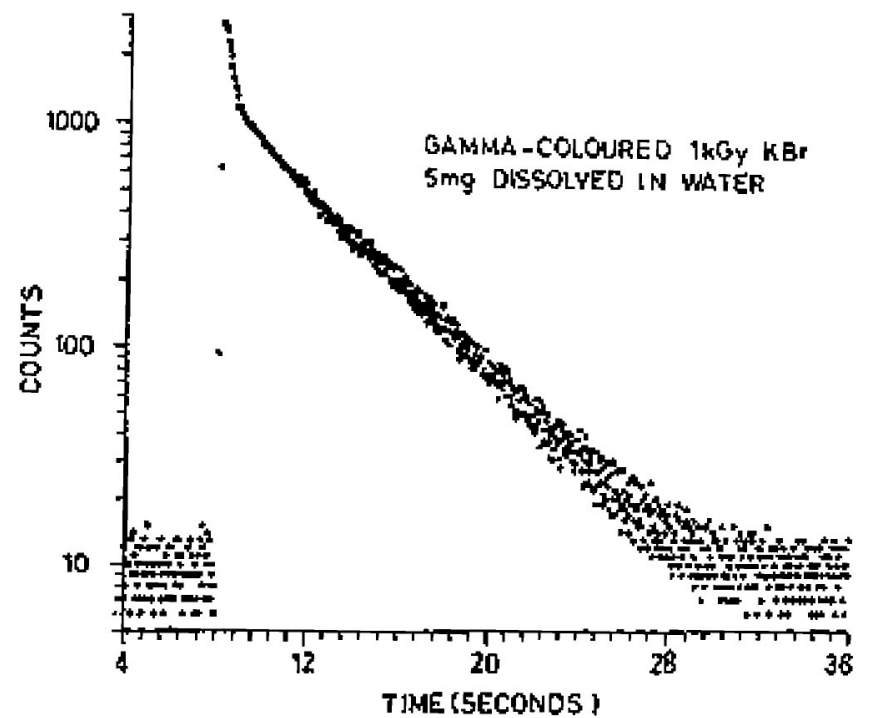

FIG. 2. Experimental lyoluminescent spectrum, recorded online for $\mathrm{KCl}$ (Ref. 16).

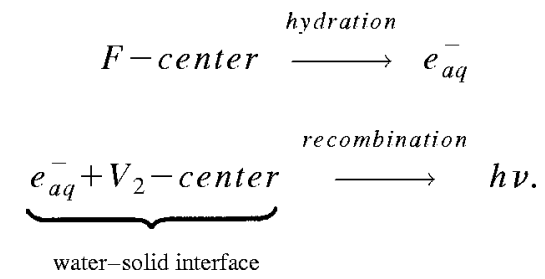

The increase in luminosity of colored halide crystals is due to the presence of trace impurities in these crystals. The released $F$ centers on hydration recombine with the acceptor impurities $(I)$ at the water-solid interface:

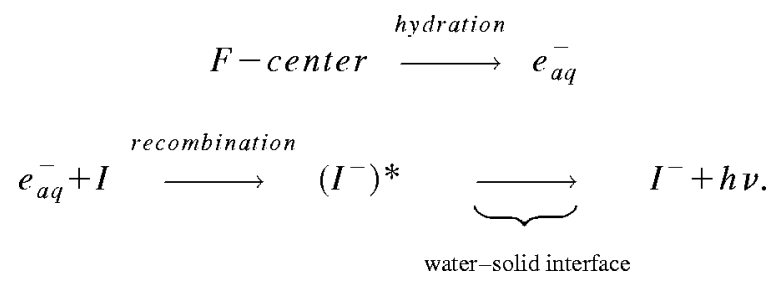

\section{THEORY}

The parameters considered in our theory of the LL intensity are the same as those mentioned in Refs. 6 and 15.

When the $\gamma$-irradiated halide crystal containing $N$ molecules at any time $t$ is dissolved in a given volume of the solvent, it dissolves in the solvent at the specific rate $\alpha$. Then the rate of decrease in the number of solute atoms may be written as

$$
-\frac{d N}{d t}=\alpha N .
$$

The rate of LL depends not only upon this rate of dissolution but also upon the extent of a priori exposure of the crystal to ionizing radiation. At first the LL intensity is seen to increase with the radiation dose and then attains a saturation value for larger radiation doses. Now working in this saturation zone, we easily see that our theory becomes independent of the radiation dose. So neglecting this effect and taking $N=N_{0}$ at $t=0$, integration of Eq. (1) gives

$$
N=N_{0} e^{-\alpha t} .
$$

Assuming a linear dependence of the rate $R$ of generation of the hydrated electrons on $n_{F}$, the density of $F$ centers, and again considering $R$ proportional to $-d N / d t$, we get

$$
\begin{gathered}
R=\Gamma n_{F}\left(-\frac{d N}{d t}\right), \\
\text { i.e., } R=\Gamma n_{F} \alpha N_{0} e^{-\alpha t},
\end{gathered}
$$

where $\Gamma$ is a factor defining the correlation of the number of hydrated electrons with the number of dissolved color centers of the crystal.

Now the microscopic dynamics of the system will set a competition between the increase in the number of hydrated electrons due to the dissolution of the crystal in the solvent and the decrease in the same number of electrons due to recombination of hydrated electrons with holes at the crystal surface. Since a hole recombines with an electron, if there are $n$ number of hydrated electrons at any time $t$, a hole has $n$ possible opportunities to combine with these $n$ electrons. Since there will also be $n$ holes at the surface, these $n$ holes can combine with the $n$ electrons in $n^{2}$ possible ways.

Then the rate equation becomes

$$
\frac{d n}{d t}=R-\left(\sigma_{r} N_{r} v\right) n^{2}
$$

where $\sigma_{r}$ is the capture cross section of the holes, $N_{r}$ is the density of the recombination centers, i.e., holes, and $v$ is the average drift velocity of the hydrated electrons.

Taking $\beta=\sigma_{r} N_{r} v$ as the rate constant for the recombination of hydrated electrons with holes, the rate equation now turns out to be

$$
\frac{d n}{d t}=\Gamma \alpha n_{F} N_{0} e^{-\alpha t}-\beta n^{2}
$$

The above differential equation being a nonlinear one with no exact analytical solution, we first check for the stability of the system from the standpoint of dynamical systems and then obtain an estimate of the solutions in the asymptotic limit. Finally we compare all these results with exact numerically integrated solutions of Eq. (5) for a range of characteristic values of the parameters.

\section{A. Dynamical system study}

From Eq. (5), we see that

$$
\dot{n}(t)=\lambda \alpha e^{-\alpha t}-\beta n^{2},
$$

where $\lambda=\Gamma n_{F} N_{0}$. Since Eq. (6) is a nonautonomous system of dimension 2, we can reduce it to an equivalent description of two autonomous systems by a simple variable redefinition.

For this we put $Z=e^{-\alpha t}$. Thus the equivalent autonomous description looks like

$$
\dot{n}(t)=\lambda \alpha Z-\beta n^{2},
$$




$$
\dot{Z}(t)=-\alpha Z \text {. }
$$

The obvious fixed point is $Z=n=0$. Thereafter simple linear stability analysis around this fixed point gives us the eigenvalues of the stability matrix as 0 and $-\alpha$. The very existence of this marginal value (zero) renders any comment about the stability of the system untenable in the limit of large time scales. The way out is to look for the asymptotic variation of $n(t)$ with $t$ in the limits of small and large time scales. The solution of Eq. (5) at small times looks like

$$
\left.n(t)\right|_{t \rightarrow 0}=\sqrt{\frac{\lambda \alpha}{\beta}}\left(\frac{N_{0}^{\prime}-1}{N_{0}^{\prime}+1}\right)\left[1+\frac{4 N_{0}^{\prime}}{N_{0}^{\prime 2}-1} \sqrt{\lambda \alpha \beta} t\right],
$$

where $N_{0}^{\prime}$ depends upon $n_{0}$, the initial value of $n(t)$, and is given by

$$
N_{0}^{\prime}=\frac{\sqrt{\frac{\lambda \alpha}{\beta}}+n_{0}}{\sqrt{\frac{\lambda \alpha}{\beta}}-n_{0}} .
$$

The above equation shows that when solute is added to the solvent, the initial LL intensity is a linear function of time [since LL intensity is proportional to $n(t)$ ], characterized by an initial non-zero radiation, which is a function of $N_{0}^{\prime}$. This result is in exact harmony with experiments [and accidentally with Eq. (14) of B. P. Chandra et al., ${ }^{15}$ although the proportionality constants differ and also no initial nonzero radiation could be found in Ref. 15]. At large times Eq. (5) can be solved to give

$$
\left.n(t)\right|_{t \rightarrow \infty}=\frac{n_{0}}{1+n_{0} \beta t}
$$

which is the equation of a shifted rectangular hyperbola and conforms well in this limit with the numerically solved glow curves (Fig. 1) for the whole range of the parameter values.

\section{B. Numerical analysis}

The method of numerical integration employed here is the standard Runge-Kutta (order IV) with characteristic values of the parameters $\gamma, \alpha, n_{F}, n_{0}$, and $\beta$. Figure 1 shows plots of $n(t)$ against the time $t$ for a whole set of parameter values. These are seen to have exactly the same shape as the experimental curve in Fig. 2. Also it is easy to see that similar curves can be generated on extrapolation of the asymptotic solutions of $n(t)$, since Eq. (6) is a regular equation and hence is not expected to show any discontinuous jumps. Changing the characteristic values of the parameters does not in any way change the nature of the curve, as is evident from the asymptotic zone analysis. Simple rescaling of $n(t)$ and $t$ gives back essentially the same curves as in Refs. 16 and 17 confirming our analytical arguments.

In order to compare our theory with some other important experimental observations, we write down an analytical solution exact up to the second order in Picard's mathod, which is generally valid for not so large $t$ and $n(t=0)=0$ (although at $t=0$, a small value of $n(t=0)$ indeed exists):

$$
n(t)=\left(\lambda+\frac{2 \beta \lambda^{2}}{\alpha}\right)\left(1-e^{-\alpha t}\right)-\beta \lambda^{2} t-\frac{\beta \lambda^{2}}{2 \alpha}\left(1-e^{-2 \alpha t}\right) .
$$

Since glow curves obtained from this solution, at small times, tally reasonably well with the curves obtained from exact numerical integration of Eq. (5) (Fig. 1), we are in a position now to compare some other experimental facts from the above second-order solution. Also the order of magnitude calculations of the maxima in the LL intensity curves (which essentially appear close to $t=0$ ) from this approximate solution $(\sim 1$ theoretical unit) conforms well with the numerical values. For small times, the above solution agrees well with the asymptotic result shown previously. Since the rate of dissolution of the solute in the solvent is slow compared to the rate of recombination of hydrated electrons with holes, $\beta \gg \alpha$. Also from experiment we know that $n_{F} \sim 10^{15}-10^{18}$ (Ref. 18).

Utilizing the above facts in Eq. (12), we see that as $\alpha$ increases with temperature, the LL intensity $[\propto n(t)]$ should also increase with temperature which can also apparently be seen from the asymptotic study.

\section{COMPARISON WITH EXPERIMENTS}

A detailed measurement of most of the LL properties have been made in the past, some of which conform well to our theory presented here.

The nature of the decay curves as seen by Banerji et al. ${ }^{16}$ [Fig. 2], qualitatively matches not only with the exact numerically integrated theoretical curve but also with the second-order approximate analytic solution (Picard's solution). The asymptotic solutions of the equation at large times are also in conformity with the basic nature of these curves. In all these plots the LL intensity is seen to fall rapidly at first and then the decay portion shows a more moderate steady decrease. However, at the initial instant, the curve is almost linear, and the intensity increases as the temperature of the solution rises. This temperature dependence holds for all times, although thermal bleaching of $F$ centers takes place at higher temperatures and thereafter the LL intensity is seen to decrease, a fact which we cannot establish from our simple theory.

An early effort towards the developement of an analytical theory for the LL intensity characteristics was due to B. P. Chandra et al. ${ }^{15}$ They attempted the development of a rate equation in the same line as Chatterjee, Sur, and Roy ${ }^{9}$ for alkali halides utilizing the mechanism due to Atari ${ }^{14}$ which happened to be our basis too. However, they failed to account for the recombination of all the $n$ holes with all the $n$ number of electrons giving rise to $n^{2}$ combinations. Consequently, the linear theory developed by them, although solvable exactly, failed to reproduce the experimental curves as seen in Refs. 16 and 17 and as shown in Fig. 2. While their theory could accidentally explain the temperature dependence of the LL intensity, as observed in the experiments, the detailed variation of the lyoluminescent intensity with time could not be accounted for due to the absence of the dominant nonlinearity in the system. We have overcome all these drawbacks in our theory, which is quite capable of explaining these important experimental observations.

In conclusion, it would be relevant to add that we started 
TABLE I. Experimental vs theoretical decay times: sample weight for experimental $\sim 5 \mathrm{mg}$, solvent: water, $p \mathrm{H}$ : 6.5 , oxygen content: 9 ppm (Ref. 16), theoretical parameters: $\alpha=1, \lambda=1$.

\begin{tabular}{|c|c|c|c|c|c|}
\hline \multirow[b]{2}{*}{ Sample } & \multicolumn{4}{|c|}{ Expt. decay times (s) } & \multirow[b]{2}{*}{$\begin{array}{c}\text { Theor. decay } \\
\text { times (s) }\end{array}$} \\
\hline & $\begin{array}{c}\text { Dose in } \\
\text { kGy }\end{array}$ & Short & Long & $\beta$ & \\
\hline & 0.1 & $0.36 \pm 0.08$ & $4.35 \pm 0.60$ & & \\
\hline \multirow[t]{4}{*}{$\mathrm{KBr}$} & 0.5 & $0.43 \pm 0.08$ & $4.24 \pm 0.58$ & 0.6 & 4.3 \\
\hline & 1.0 & $0.40 \pm 0.06$ & $4.32 \pm 0.50$ & & \\
\hline & 2.0 & $0.42 \pm 0.06$ & $4.28 \pm 0.45$ & & \\
\hline & 0.1 & $0.25 \pm 0.06$ & $2.35 \pm 0.40$ & & \\
\hline \multirow[t]{3}{*}{$\mathrm{KCl}$} & 0.5 & $0.18 \pm 0.06$ & $2.20 \pm 0.38$ & 1.5 & 2.3 \\
\hline & 1.0 & $0.20 \pm 0.04$ & $2.30 \pm 0.30$ & & \\
\hline & 2.0 & $0.23 \pm 0.04$ & $2.28 \pm 0.28$ & & \\
\hline
\end{tabular}

with an objective of developing a theory of lyoluminescence of alkali halide crystals. In the process we landed up with a nonlinear rate equation whose exact numerical and approximate analytical solutions are capable of explaining qualitatively the temperature dependence and the overall time dependence of the LL intensity. Although we have been able to investigate and reconcile with experiments some of the properties of lyoluminescence, much more remains unscathed by our elementary theory. Notable among them are variation of peak heights with irradiation dose, peak heights vs concentration and $p \mathrm{H}$ of solution, etc. In our pursuit of simplification, we have neglected the corresponding parameters controlling these effects, which could possibly have shed light on a few other experimental results. Accordingly, we hope to develop a complete theory of alkali halide crystals in future including all the relevant parameters and the solution of the apparently cumbersome equation generated therefrom should be able to reproduce the experimental results in detail.

It seems worthwhile to mention here that since in drawing the theoretical graphs, we have utilized values of $\alpha$ and $\beta$ in units of $\lambda$ (which hence is always taken to be unity), exact quantitative comparison between our theoretical graphs with the experimental ones would have required a suitable normalization of our units used with the real physical units. Once this is done, curve-fitting procedures could be employed to determine the exact values of $\alpha, \beta$, and $\lambda$, following which an exact measure of the decay times could be done theoretically. Two typical examples have been shown in Table I, where for characteristic values of the theoretical parameters used ( $\alpha, \beta$, and $\lambda$ ), the long components of the decay time match exactly with the experimental values. The values of the short components could not be resolved theoretically, which is understandable, since the experimental observations show that the small values of decay times of the short components of the spectrum fall well within the fluctuation range of the long components. We plan the exact enumeration of decay times as a future project.

\section{ACKNOWLEDGMENT}

A.K.C. and P.C. acknowledge partial financial support by the C.S.I.R., India. Also A.K.C. is pleased to acknowledge his many illuminating discussions with Professor Jayanta K. Bhattacharjee and encouragements from K. S. Das and Dr. Manabesh Bhattacharyya throughout the course of this work.

${ }^{1}$ E. Wiedemann and G. C. Schmidt, Ann. Phys. (Leipzig) 56, 210 (1895).

${ }^{2}$ G. Ahnstrom and G. V. Ehrenstein, Acta Chem. Scand. 13, 109 (1959); 13, 855 (1959).

${ }^{3}$ G. Ahnstrom and L. Ehrenberg, RISO Rep. 16, 15 (1960).

${ }^{4}$ G. Ahnstrom, Acta Chem. Scand. 19, 300 (1965).

${ }^{5}$ B. Lelievre and J. P. Adloff, J. Phys. (France) 25, 789 (1964).

${ }^{6}$ G. T. Reynolds, J. Lumin. 54, 43 (1992).

${ }^{7}$ W. G. Burns and T. F. Williams, Nature (London) 173, 1043 (1955).

${ }^{8}$ J. P. Mittal (unpublished).

${ }^{9}$ B. K. Chatterjee, T. Sur, and S. C. Roy, Phys. Rev. B 47, 555 (1993).

${ }^{10}$ K. V. Ettinger and K. J. Puite, Int. J. Appl. Radiat. Isot. 33, 1115 (1982).

${ }^{11}$ K. J. Puite and K. V. Ettinger, Int. J. Appl. Radiat. Isot. 33, 1139 (1982).

${ }^{12}$ G. A. Russel, J. Am. Chem. Soc. 79, 3871 (1957).

${ }^{13}$ R. F. Vassil'ev, Prog. React. Kinet. 4, 305 (1967).

${ }^{14}$ N. A. Atari, J. Lumin. 21, 305 (1979).

${ }^{15}$ B. P. Chandra, R. K. Tiwari, Reenu Mor, and D. P. Bisen, J. Lumin. 75, 127 (1997).

${ }^{16}$ P. Banerji, H. K. Kundu, D. Banerjee, and R. Bhattacharyya, J. Lumin. 62, 109 (1994).

${ }^{17}$ C. D. Kalkar, Radiat. Phys. Chem. 34, 729 (1989).

${ }^{18}$ T. Sur, Ph.D. thesis, Jadavpur University, 1991. 